\title{
5G Technology : A Survey of Architecture and Emerging Technologies
}

\author{
Dr. Hitesh H Vandra
}

Shri J M Sabva Institute of Engineering and Technology, Botad, Gujarat, India

\begin{abstract}
Research on 5G mobile wireless technologies has been very active in both academia and industry in the past few years. While there has been certain consensus on the overall requirements of $5 \mathrm{G}$ wireless systems (e.g., in data rate, network capacity, delay), various enabling wireless technologies have been considered and studied to achieve these performance targets. It has been quite clear, however, that there would be no single enabling technology that can achieve all diverse and even conflicting $5 \mathrm{G}$ requirements.

In the near future, i.e., beyond $4 \mathrm{G}$, some of the prime objectives or demands that need to be addressed are increased capacity, improved data rate, decreased latency, and better quality of service. To meet these demands, drastic improvements need to be made in cellular network architecture.

This paper presents the results of a detailed survey on the fifth generation (5G) cellular network architecture and some of the key emerging technologies that are helpful in improving the architecture and fulfil the demands. In this detailed survey, the prime focus is on the $5 \mathrm{G}$ cellular network architecture, massive multiple input multiple output technology, and device-to-device communication (D2D). Along with this, some of the emerging technologies that are addressed in this paper include interference management, spectrum sharing with cognitive radio, ultra-dense networks, multi-radio access technology association, full duplex radios, millimeter wave solutions for $5 \mathrm{G}$ cellular networks, and cloud technologies for $5 \mathrm{G}$ radio access networks and software defined networks. In this paper, a general probable $5 \mathrm{G}$ cellular network architecture is proposed, which shows that D2D, small cell access points, network cloud, and the Internet of Things can be a part of5G cellular network architecture. A detailed survey is included regarding current research projects being conducted in different countries by research groups and institutions that are working on $5 \mathrm{G}$ technologies.
\end{abstract}

Keywords : 5G, cloud, D2D, massive MIMO, mm-wave, relay, small-cell 5G wireless, 5G waveform, Dense networks, Het Nets, Massive MIMO, C-RAN, Software defined networking

\section{INTRODUCTION}

Research and development activities on 5G mobile wire-less technologies have attracted lots of interest in both academia and industry worldwide over the last several years. These initiatives have been driven by many evidences that the currently deployed 4G wireless network could not provide satisfactory support in the near future. In particular, the mobile data traffic has been exploding and this trend is expected to continue in the coming years. It is predicted that the $5 \mathrm{G}$ wireless system should be able to deliver as much as 1000 times of the capacity provided by today's mobile networks. Moreover, many emerging broadband wireless applications such as high-resolution video streaming and tactile Internet demand much higher data rates, which could be 10 100 times of what is offered by $4 \mathrm{G}$ wireless networks. 
Recent technology constituent like high-speed packet access (HSPA)and long-term evolution (LTE) will be launched as a segment of the advancement of current wireless based technologies. Nevertheless, auxiliary components may also constitute future new wireless based technologies, which may adjunct the evolved technologies. Specimen of these new technology components are different ways of accessing spectrum and considerably higher frequency ranges, the instigation of massive antenna configurations, direct device-to-device communication, and ultra-dense deployments.

This paper presents our view on the future of wireless communication for 2020 and beyond. In this paper, we describe the key challenges that will be encountered by future wireless communication while enabling the networked society. Along with this, some technology routes that may be taken to full-fill these challenges.

\section{EVOLUTION OF WIRELESS TECHNOLOGIES}

The evolution of wireless begins here and is shown in Fig. 1. It shows the evolving generations of wireless technologies in terms of data rate, mobility, coverage and spectral efficiency. As the wireless technologies are growing, the data rate, mobility, coverage and spectral efficiency increases. It also shows that the $1 \mathrm{G}$ and $2 \mathrm{G}$ technologies use circuit switching while $2.5 \mathrm{G}$ and $3 \mathrm{G}$ uses both circuit and packet switching and the next generations from $3.5 \mathrm{G}$ to now i.e. $5 \mathrm{G}$ are using packet switching. Along with these factors, it also differentiate between licensed spectrum and unlicensed spectrum. All the evolving generations use the licensed spectrum while the WiFi, Bluetooth and WiMAX are using the unlicensed spectrum. An overview about the evolving wireless technologies is below:

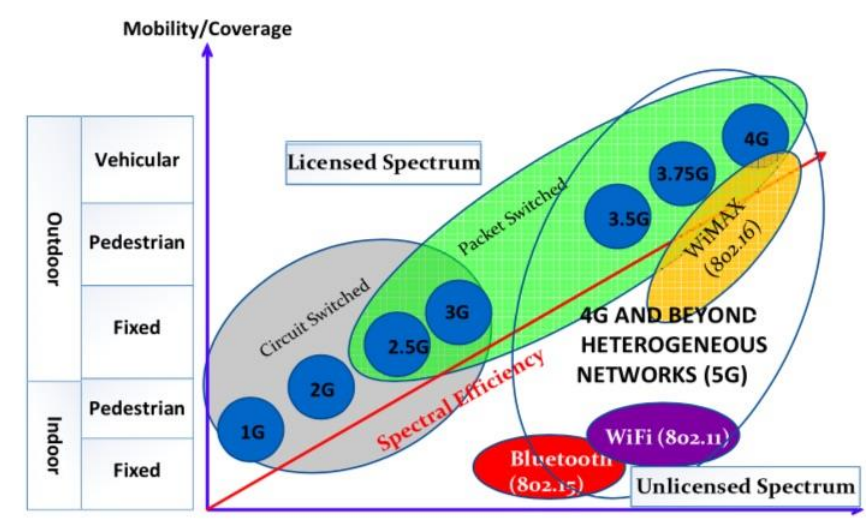

Figure 1. Evolution of wireless technologies.

A. $1 \mathrm{G}$

The 1st generation was announced in initial 1980's. It has a data rate up to $2.4 \mathrm{kbps}$. Major subscribers were Advanced Mobile Phone System (AMPS), Nordic Mobile Telephone (NMT), and Total Access Communication System (TACS). It has a lot of disadvantages like below par capacity, reckless handoff, inferior voice associations, and with no security, since voice calls were stored and played in radio towers due to which vulnerability of these calls from unwanted eavesdropping by third party increases [7]. The first generation of mobile network was deployed in Japan by Nippon Telephone and Telegraph company (NTT) in Tokyo during 1979. In the beginning of 1980s, it gained popularity in the US, Finland, UK and Europe. This system used analogue signals and it had many disadvantages due to technology limitations.

B. $2 \mathrm{G}$

The 2nd generation was introduced in late 1990's. Digital technology is used in $2^{\text {nd }}$ generation mobile telephones. Global Systems for Mobile communications (GSM) was the first $2^{\text {nd }}$ generation system, chiefly used for voice communication and having a data rate up to $64 \mathrm{kbps}$. $2 \mathrm{G}$ mobile handset battery lasts longer because of the radio signals having low power. It also provides services like Short Message Service (SMS) and e- 
mail. Vital eminent technologies were GSM, Code Division Multiple Access (CDMA), and IS-95 in references. Second generation of mobile communication system introduced a new digital technology for wireless transmission also known as Global System for Mobile communication (GSM). GSM technology became the base standard for further development in wireless standards later. This standard was capable of supporting up to 14.4 to $64 \mathrm{kbps}$ (maximum) data rate which is sufficient for SMS and email services. Code Division Multiple Access (CDMA) system developed by Qualcomm also introduced and implemented in the mid 1990s. CDMA has more features than GSM in terms of spectral efficiency, number of users and data rate.

\section{C. $2.5 \mathrm{G}$}

It generally subscribes a 2nd generation cellular system merged with General Packet Radio Services (GPRS) and other amenities doesn't commonly endow in $2 \mathrm{G}$ or $1 \mathrm{G}$ networks. A $2.5 \mathrm{G}$ system generally uses $2 \mathrm{G}$ system frameworks, but it applies packet switching along with circuit switching. It can assist data rate up to $144 \mathrm{kbps}$. The main $2.5 \mathrm{G}$ technologies were GPRS, Enhanced Data Rate for GSM Evolution (EDGE), and Code Division Multiple Access (CDMA) 2000. In order to support higher data rate, General Packet Radio Service (GPRS) was introduced and successfully deployed. GPRS was capable of data rate up to $171 \mathrm{kbps}$ (maximum). EDGE - Enhanced Data GSM Evolution also developed to improve data rate for GSM networks. EDGE was capable to support up to 473.6kbps (maximum). Another popular technology CDMA2000 was also introduced to support higher data rate for CDMA networks. This technology has the ability to provide up to $384 \mathrm{kbps}$ data rate (maximum).

\section{D. $3 \mathrm{G}$}

The $3^{\text {rd }}$ generation was established in late 2000 . It imparts transmission rate up to $2 \mathrm{Mbps}$. Third generation $(3 \mathrm{G})$ systems merge high speed mobile access to services based on Internet Protocol (IP). Aside from transmission rate, unconventional improvement was made for maintaining QoS. Additional amenities like global roaming and improved voice quality made $3 \mathrm{G}$ as a remarkable generation. The major disadvantage for $3 \mathrm{G}$ handsets is that, they require more power than most $2 \mathrm{G}$ models. Along with this $3 \mathrm{G}$ network plans are more expensive than $2 G$ as per references. Since $3 G$ involves the introduction and utilization of Wideband Code Division Multiple Access (WCDMA), Universal Mobile Telecommunications Systems (UMTS) and Code Division Multiple Access (CDMA) 2000 technologies, the evolving technologies like High Speed Uplink/Downlink Packet Access (HSUPA/HSDPA) and EvolutionData Optimized (EVDO) has made an intermediate wireless generation between $3 \mathrm{G}$ and $4 \mathrm{G}$ named as $3.5 \mathrm{G}$ with improved data rate of 5-30 Mbps.

\section{E. $3.75 \mathrm{G}$}

Long-Term Evolution technology (LTE) and Fixed Worldwide Interoperability for Microwave Access (WIMAX) is the future of mobile data services. LTE and Fixed WIMAX has the potential to supplement the capacity of the network and provides a substantial number of users the facility to access a broad range of high speed services like on demand video, peer to peer file sharing and composite Web services. Along with this, a supplementary spectrum is accessible which accredit operators manage their network very compliantly and offers better coverage with improved performance for less cost.

\section{F. $4 \mathrm{G}$}

$4 \mathrm{G}$ is generally referred as the descendant of the $3 \mathrm{G}$ and 2G standards. 3rd Generation Partnership Project (3GPP) is presently standardizing Long Term Evolution (LTE) Advanced as forthcoming 4G standard along with Mobile Worldwide Interoperability for Microwave Access (WIMAX). A 4G system improves the prevailing communication networks by imparting a complete 
and reliable solution based on IP. Amenities like voice, data and multimedia will be imparted to subscribers on every time and everywhere basis and at quite higher data rates as related to earlier generations. Applications that are being made to use a 4G network are Multimedia Messaging Service (MMS), Digital Video Broadcasting (DVB), and video chat, High Definition TV content and mobile TV.

G. $5 \mathrm{G}$

$5 \mathrm{G}$ will be using advanced technologies to deliver ultra-fast internet and multimedia experience for customers. Current LTE advanced networks will transform into supercharged $5 \mathrm{G}$ networks in future. In order to achieve higher data rate, $5 \mathrm{G}$ technology will use millimetre waves and unlicensed spectrum for data transmission. Complex modulation technique has been developed to support massive data rate for Internet of Things.

To contemplate $5 \mathrm{G}$ network in the market now, it is evident that the multiple access techniques in the network are almost at a still and requires sudden improvement. Current technologies like OFDMA will work at least for next 50 years. Moreover, there is no need to have a change in the wireless setup which had come about from $1 \mathrm{G}$ to $4 \mathrm{G}$. Alternatively, there could be only the addition of an application or amelioration done at the fundamental network to please user requirements. This will provoke the package providers to drift for a $5 \mathrm{G}$ network as early as $4 \mathrm{G}$ is commercially set up [8]. To meet the demands of the user and to overcome the challenges that has been put forward in the $5 \mathrm{G}$ system, a drastic change in the strategy of designing the $5 \mathrm{G}$ wireless cellular architecture is needed. A general observation of the researchers has shown in [14] that most of the wireless users stay inside for approximately 80 percent of time and outside for approximately 20 percent of the time. In present wireless cellular architecture, for a mobile user to communicate whether inside or outside, an outside base station present in the middle of a cell helps in communication. So for inside users to communicate with the outside base station, the signals will have to travel through the walls of the indoors, and this will result in very high penetration loss, which correspondingly costs with reduced spectral efficiency, data rate, and energy efficiency of wireless communications. To overcome this challenge, a new idea or designing techniques that has come in to existence for scheming the $5 \mathrm{G}$ cellular architecture is to distinct outside and inside setups. With this designing technique, the penetration loss through the walls of the building will be slightly reduced. This idea will be supported with the help of massive MIMO technology, in which geographically dispersed array of antenna's are deployed which have tens or hundreds of antenna units. Since present MIMO systems are using either two or four antennas, but the idea of massive MIMO systems has come up with the idea of utilizing the advantages of large array antenna elements in terms of huge capacity gains.

To build or construct a large massive MIMO network, firstly the outside base stations will be fitted with large antenna arrays and among them some are dispersed around the hexagonal cell and linked to the base station through optical fibre cables, aided with massive MIMO technologies. The mobile users present outside are usually fitted with a certain number of antenna units but with cooperation a large virtual antenna array can be constructed, which together with antenna arrays of base station form virtual massive MIMO links. Secondly, every building will be installed with large antenna arrays from outside, to communicate with outdoor base stations with the help of line of sight components. The wireless access points inside the building are connected with the large antenna arrays through cables for communicating with indoor users. This will significantly improves the energy efficiency, cell average throughput, data rate, and spectral efficiency of the cellular system but at the expense of increased infrastructure cost. With the introduction of such an architecture, the inside users will only have to connect or communicate with inside wireless access points while larger 
antenna arrays remained installed outside the buildings [8]. For indoor communication, certain technologies like Wi-Fi, Small cell, ultra-wideband, millimetres wave communications, and visible light communications are useful for small range communications having large data rates. But technologies like millimetre wave and visible light communication are utilizing higher frequencies which are not conventionally used for cellular communications. But it is not an efficient idea to use these high frequency waves for outside and long distance applications because these waves

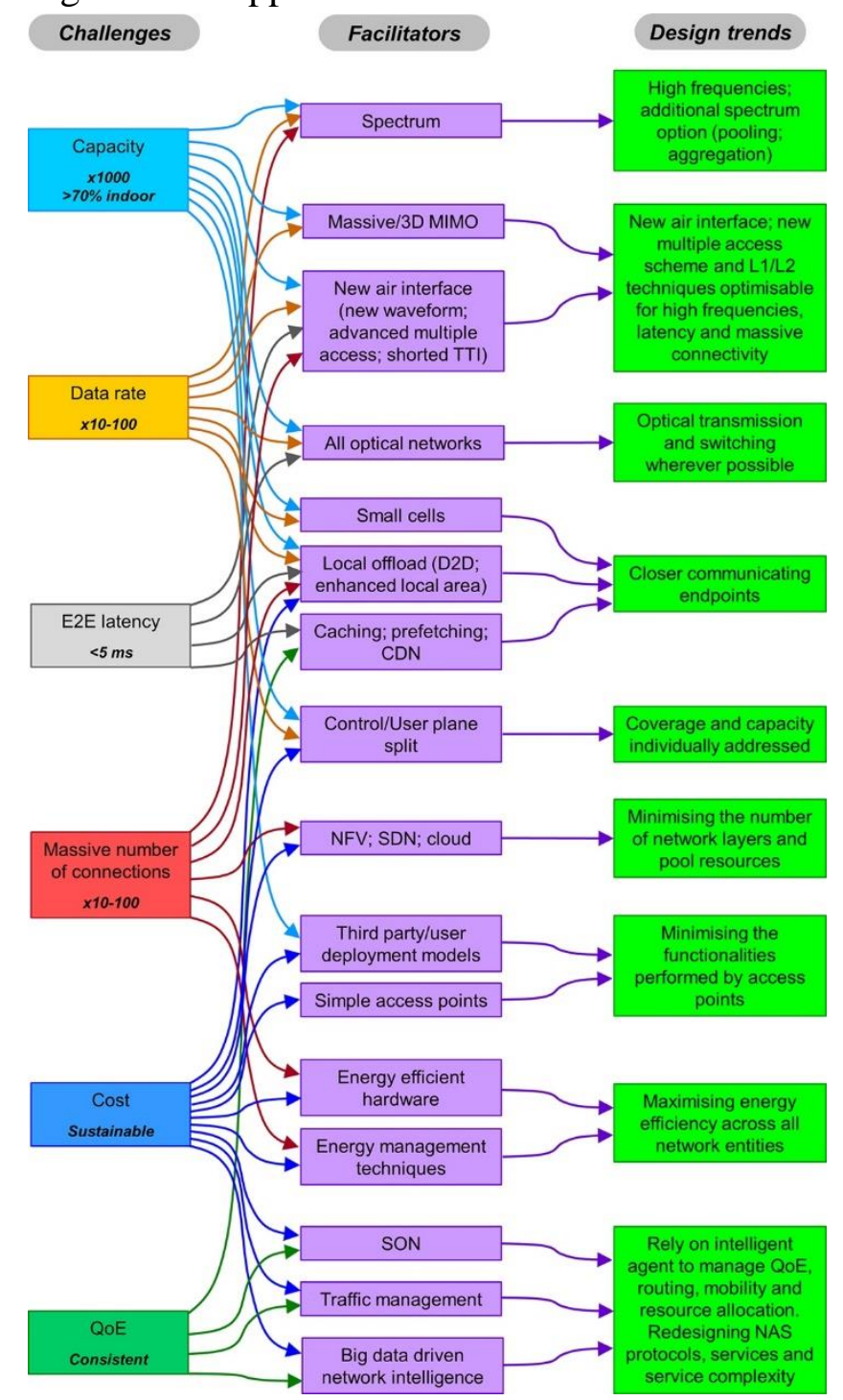

Figure 2. 5G challenge, facilitators, and design fundamental [20].

will not infiltrate from dense materials efficiently and can easily be dispersed by rain droplets, gases, and flora. Though, millimetre waves and visible light communications technologies can enhance the transmission data rate for indoor setups because they have come up with large bandwidth. Along with the introduction of new spectrum, which is not being conventionally used for wireless communication, there is one more method to solve the spectrum shortage problem by improving the spectrum utilization of current radio spectra through cognitive radio (CR) networks.

Since the 5G cellular architecture is heterogeneous, so it must include macro-cells, microcells, small cells, and relays. A mobile small cell concept is an integral part of $5 \mathrm{G}$ wireless cellular network and partially comprises of mobile relay and small cell concepts. It is being introduced to put up high mobility users, which are inside the automobiles and high speed trains. Mobile small cells are positioned inside the moving automobiles to communicate with the users inside the automobile, while the massive MIMO unit consisting of large antenna arrays is placed outside the automobile to communicate with the outside base station. According to user's opinion, a mobile small cell is realized as a regular base station and its allied users are all observed as a single unit to the base station which proves the above idea of splitting indoor and outdoor setups. Mobile small cell users have a high data rate for data rate services with considerably reduced signalling overhead, as shown in. As the $5 \mathrm{G}$ wireless cellular network architecture consists of only two logical layers: a radio network and a network cloud. Different types of components performing different functions are constituting the radio network. The network function virtualization (NFV) cloud consists of a User plane entity (UPE) and a Control plane entity (CPE) that per- form higher layer functionalities related to the User and Control plane, respectively. Special network functionality as a service (XaaS) will provide service as per need, resource pooling is one of the examples. XaaS is the connection between a radio network and a network cloud. 
The 5G cellular network architecture is explained in [8] and [20]. It has equal importance in terms of front end and backhaul network respectively. In this paper, a general 5G cellular network architecture has been proposed as shown in Fig. 3. It describes the interconnectivity among the different emerging technologies like Massive MIMO network, Cognitive Radio network, mobile and static smallcell networks. This proposed architecture also explains the role of network function virtualization (NFV) cloud in the 5G cellular network architecture. The concept of Device to Device (D2D) communication, small cell access points and Internet of things (IoT) has also been incorporated in this proposed $5 \mathrm{G}$ cellular network architecture. In general, this proposed $5 \mathrm{G}$ cellular network architecture may provide a good platform for future $5 \mathrm{G}$ standardization network.

But there are several issues that need to be addressed in order to realize the wireless network architecture in particular and 5G networks in general. Some of these issues are summarized in Table. 3.

\section{Perspectives on 5G enabling technologies and open research issues}

In this section, we provide our perspectives on the role and potential of some important $5 \mathrm{G}$ enabling technologies. We also describe in details some open research issues related to these technologies. Moreover, we will discuss how they can complement and be effectively combined to meet different performance requirements of the future $5 \mathrm{G}$ wireless network. 2.1 Waveform design for $5 \mathrm{G}$ wireless systems

\section{i. Waveform design for $\mathbf{5 G}$ wireless systems}

It has been recognized that the OFDM waveform currently adopted in the long-term evolution (LTE) standard has several limitations in supporting the $5 \mathrm{G}$ requirements. Specifically, 5G traffic is expected to have very diverse characteristics and requirements in data column, communications rate, delay, and reliability. For example, applications such as video streaming and augmented reality demand very high communication rates for realtime communications. Moreover, various emerging M2M applications such as those for smart grids have sporadic and busty traffic with small amount of data to be delivered in each transmission. Other wireless applications such as vehicular communications and robotics control require highly reliable and ultra-low delay. Finally, the number of wireless connections supporting future $\mathrm{M} 2 \mathrm{M}$ and Internet-of-Things applications is expected to be significantly larger than that due to mostly humantype communications in today's wireless networks.

The OFDM waveform possesses several desirable features including low-complexity implementation using fast Fourier transform (FFT) and inverse FFT (IFFT) blocks, simple equalization, flexibility in supporting per subcarrier adaptive modulation and MIMO communications. However, strict synchronization, which involves large signalling overhead, is required to maintain communication orthogonality and satisfactory communication quality. Moreover, the OFDM signalling requires a cyclic prefix (CP) and null guard bands at the spectrum edges, which reduce the spectral efficiency. In general, development of more spectral and power efficient waveforms com- pared to the current state-of-the-art is of great interest to support the exponential growth of mobile traffic and operation cost reduction in the future wireless system. Finally, current frame structure and implementation of various protocols such as scheduling and retransmissions in the $4 \mathrm{G}$ LTE systems offers the latency of multiple $10 \mathrm{~ms}$, which is far beyond the $5 \mathrm{G}$ strict delay requirement of 1 
ms. In fact, this $5 \mathrm{G}$ delay requirement implies that the physical-layer delay budget is about $100 \mu \mathrm{s}$.

There have been several proposals on waveforms for $5 \mathrm{G}$ wireless networks. In particular, faster-thanNyquist (FTN) signalling has recently received renewed interest since it can potentially increase the system capacity by packing more data in the time and/or frequency domains. In the time domain, this can be achieved by allowing data bearing pulses to be sent faster. While this design destroys the orthogonality, satisfactory detection performance can still be achieved by employing a sophisticated detection technique at the receiver to mitigate the intentional ISI introduced at the transmitter. In addition, employment of FTN signalling in both time and frequency domains results in multi-carrier FTN, which attains larger spectrum efficiency compared to FTN in time or frequency alone. Although some significant progresses have been achieved in recent years on under- standing FTN signalling, more research is needed to study and develop efficient designs to meet the performance and QoS requirements of $5 \mathrm{G}$ wireless systems. In particular, practical implementation aspects deserve more research and development efforts.

Filter bank multi-carrier (FBMC) is one of the $5 \mathrm{G}$ waveform candidates where per-subcarrier filtering is per- formed to suppress the sidelobes and the FFT/IFFT blocks can still be employed as in the OFDM modulation. The bandlimitedness of FBMC would enable it to offer higher spectrum efficiency than OFDM and more flexibility in exploiting white spaces in cognitive radio networks. Moreover, FBMC is more robust to synchronization and frequency misalignments compared to OFDM; therefore, FBMC would be more suitable for random access to support sporadic M2M traffic with loose synchronization requirements. Despite these advantages, FBMC is more difficult to realize the MIMO communications com- pared to OFDM. However, recent progresses on advanced physical techniques have led to efficient ways to implement
MIMO-FBMC with comparable performance with respect to MIMO-OFDM systems.

An enhanced version of FBMC, called universal filtered multi-carrier (UFMC), has been proposed recently. In UFMC, filtering is performed on the sub band basis instead of per subcarrier as in FBMC. Therefore, UFMC can still acquire the advantages of FBMC including suppression of spectral sidelobes, robustness against time and frequency synchronization. Moreover, the filter length of UFMC can be significantly shorter than that of FBMC since UFMC performs the filtering for each sub- band with larger bandwidth than subcarrier. Therefore, UFMC would be more suitable to support short-burst communications typically required by many $\mathrm{M} 2 \mathrm{M}$ applications. UFMC does not require a $\mathrm{CP}$, but using zero padding, which results in improved spectrum efficiency but becomes more sensitive to time misalignment com- pared to the existing CP-based OFDM waveform. On the other hand, the filtered OFDM (f-OFDM) technique in allows the filter length to exceed the CP length of OFDM. The fOFDM waveform can achieve a desirable frequency localization for bandwidths as narrow as a few tens of subcarriers, while keeping the intersymbol interference/inter-carrier interference (ISI/ICI) within an acceptable limit.

Another waveform design referred to as generalized frequency division multiplexing (GFDM) has been pro- posed to address the low-power, broadband, and real-time challenges of future wireless cellular networks. The GFDM aims at achieving flexible resource and QoS management by performing modulation for independent blocks each of which comprises a number of subcarriers and sub symbols. Moreover, GFDM employs filters for individual subcarriers that are circularly shifted in time and frequency. While inter-symbol interference (ISI) and inter-carrier interference (ICI) might still exist in GFDM, these undesirable effects 
can be efficiently addressed by efficient detection techniques at the receiver side.

Several 5G requirements can be met by the GFDM via appropriate tuning of different waveform parameters. The per-subcarrier filtering adopted by GFDM leads to small out-of-band emission; therefore, dynamic spectrum access of fragmented spectrum in cognitive radio scenarios can be achieved. Moreover, GFDM design allowing to flexibly configure the time-frequency blocks, which enables us to meet the stringent delay constraints of various $5 \mathrm{G}$ applications such as tactile internet and many wireless control applications. In addition, it is possible to add small cyclic prefix and suffix for an entire block to relax the time synchronization requirement of emerging $\mathrm{M} 2 \mathrm{M}$ applications while still maintaining improved spectral efficiency with respect to the OFDM waveform. In fact, most synchronization algorithms developed for OFDM remain applicable to the GFDM waveform. The paper in our special issue demonstrates how space time coding can be combined with the GFDM waveform in the multi-antenna wireless system while still allowing flexible resource sharing among users by using wireless scheduling.

There are many open challenges to address regarding the $5 \mathrm{G}$ waveform, frame structure, and related design issues in the physical layer and higher layers of future $5 \mathrm{G}$ wireless systems. One such frame structure to sup- port the $5 \mathrm{G}$ diverse QoS and traffic requirements has been proposed in. To enhance the multiple access gain, nonorthogonal multiple access schemes have been proposed. For example, the NOMA framework utilizes power-domain superposition multiplexing. In the code domain, the sparse code multiple access (SCMA) is presented in. Data symbols of users are mapped to multi-dimensional sparse codewords. Codewords are non-orthogonal but are systematically designed to allow low-complexity detection. Moreover, while a rich literature on adaptive resource allocation techniques has been developed for OFDM wireless systems over the past decades, significant more research on medium access, resource management, and higher layer design aspects to better understand the pros and cons of different wave- form alternatives is expected in the coming years. Such studies for relevant $5 \mathrm{G}$ deployment scenarios will be especially useful.

In general, there are several aspects one must consider in selection of a waveform for a communication system such as bandwidth efficiency, scalability, interoperability, implementation complexity and robustness to imperfections including non-linearity, phase noise. The final decision on a waveform hinges on a tradeoff between these often contradictory requirements. Another important factor that must be considered for waveform design and selection is the operation frequency. As the current trend in 5G development indicates, there could be two different operating frequency regions for $5 \mathrm{G}$ systems, namely below 6 $\mathrm{GHz}$ and mmWave bands. As each band could be employed for different deployment scenarios and they would exhibit different propagation properties, $5 \mathrm{G}$ systems could rely on two different waveforms for operation in the two bands.

\section{ii. Ultra-dense wireless networks exploiting microwave and mmwave communications}

Ultra-dense deployment of small cells, relays, distributed antenna systems operating on different frequency bands (e.g., microwave and mmWave) based on multiple RATs in a multi-tier heterogeneous network (HetNet) architecture provides a fundamental way to meet the capacity demand of future $5 \mathrm{G}$ wireless networks [48-50]. This heterogeneous network architecture also enables efficient traffic offloading among different network tiers and RATs to better support the growing mobile traffic with enhanced QoS, communication rates, and energy efficiency [5156]. 
In fact, research on multi-tier HetNets where communications among different network tiers (e.g., macro, micro and small-cell tiers) share the same microwave spectrum has been one of the most active research topics over the past years. One very important research theme in this direction is interference management, which can be per-formed using time, frequency, spatial, and power control approaches. Three papers published in our special issue address different analysis and design issues for wireless HetNets. Specifically, the outage analysis for cognitive HetNets is performed by using the stochastic geometry technique in. Moreover, the work proposes enhanced inter-cell interference coordination (eICIC) techniques for the two-tier HetNet where the optimal cell range expansion bias and almost blank sub- frame rate are obtained to achieve the fair resource sharing and interference management between two network tiers. The paper studies different interference management solutions for the multi-antenna moving network where it shows that the high performance moving net- work can be indeed realized with minimal impacts on the regular outdoor communications.

Other important research issues for multi-tier HetNets include mobility management and load balancing via dynamic BS association and admission control. To effectively manage cross-tier interference and perform load balancing in the dense HetNet, distributed solutions using the selfoptimization network (SON) techniques are desirable, which typically require reliable and lowdelay backhauls for a large number of small cells (i.e., pico and femto cells). High-speed backhauls are also critical to exchange the large volume of data between small cells and the core network. While low-cost backhauls using existing DSL can be used for indoor femtocells, this solution would not lead to a satisfactory QoS and performance. Indeed, backhauls remain to be one of the most critical issues, which hinders the successful widespread adoption of the dense small-cell network.

Future dense HetNets should support different types of nodes and communications. In particular, deviceto-device (D2D) communications exploiting the cellular spectrum to establish direct high-speed and reliable communications among wireless devices is an important technology. D2D communications can attain different benefits including enhancement of spectrum and energy efficiency, improving communication delay and reliability, reduction of traffic in the core network, and support of various emerging wireless applications such as public safety, proximity-based social networks, and vehicle-to- vehicle communications. To achieve these benefits, efficient radio resource management techniques for D2D communications must be developed. In, the interference alignment technique is employed jointly with user clustering and resource allocation for efficient spectrum sharing and interference management while the work proposes a joint power control and resource allocation framework for spectrum sharing between D2D and uplink cellular communications in the LTE-based wireless network.

MmWave communications has received tremendous interest from both academia and wireless industry recently since mmWave spectrum would provide a unique solution to solve the capacity requirements of the future wireless network. Moreover, the ultra-dense wireless network is a perfect choice to exploit the huge spectrum available in the mmWave frequency since the high attention in these frequency bands limits the cell size within a few hundred meters. Importantly, mmWave could potentially help solve the spectrum shortage problems for both wireless access and backhauls. In fact, the distinction between wireless access and back- haul links would become very blur in the future ultra- dense wireless network where an extremely large number of wireless connections would be expected to support 
emerging wireless $\mathrm{M} 2 \mathrm{M}$ and Internet-of-Things applications. It remains an open challenge to develop efficient physical layer techniques to achieve high-speed communications and advanced radio resource management solutions to support the joint wireless access and backhaul communications.

These potential benefits of mmWave communications for ultra-dense wireless deployment are not available without many challenges to overcome. In fact, mmWave communications require to employ large-scale antennas at both transmitter and receiver sides to provide the sufficient antenna gain to offset the high attention in this frequency regime. Toward this end, development of cost-efficient hardware architecture together with suit- ably designed channel estimation, beamforming, as well as other communications and signal processing techniques require much further research. The cost-efficient large-scale MIMO architecture where the number of RF chains is smaller than the number of antennas has been considered very potential for mmWave communications. Here, the development of effective channel estimation and hybrid beamforming solutions for this architecture using phase shifters and analog-to-digital converters (ADCs) with limited resolution presents major research challenges. Moreover, multi user and multi- cell resource allocation and beamforming design for this hybrid architecture to achieve highspeed communications deserves much more research efforts. Preliminary results along this direction have been presented recently in where the joint hybrid beamforming and scheduling solution is proposed.

As previously mentioned, mmWave could potentially provide unique solutions for Gigabit wireless access and backhaul communications. Since wireless backhaul links can be relatively static, adaptive algorithms for beam alignment to maintain reliable communications can be developed to compensate for the base station (BS) movements due to strong wind for example. Engineering adaptive beam update algorithms for large-scale MIMO systems to support mobile users is obviously more challenging since the MIMO beam width is quite narrow with the large number of antennas. Furthermore, it is expected that both single-hop and multi-hop backhaul communication solutions would be needed to deal with different communication scenarios in the future dense wireless network. Toward this end, algorithms in different layers of the protocol stack including beamforming, resource allocation, and routing must be designed to support the wireless access and multi-hop backhaul communications.

Finally, novel connection and mobility management strategies must be proposed to effectively deal with the unique characteristics of the heterogeneous mmWave dense networks such as susceptibility to blockage of mmWave links, different user velocities, and QoS requirements. Given that the mmWave communications links are very sensitive to blockage, it is likely that mobile users must maintain dual communications with both macro-BS and small-cell BS using mmWave and microwave frequency bands, respectively, to maintain continuous and reliable communications. Here, communications with a nearby small-cell BS on the mmWave spectrum can be employed to achieve high-speed data delivery whenever possible but control information can be delivered through the macro-BS using more reliable microwave communications. The split of control and data planes deems necessary and presents the unique design choice for mmWave communications. Moreover, the research on efficient BS association to achieve load balancing and reliable and highspeed communications must be rethought. It is also obvious that mobility management and admission control design for the mmWave-based dense HetNet deserve much more research before one can indeed bring potential benefits of mmWave communications to practice. 


\section{iii. Massive and full-dimension MIMO technologies}

While the MIMO technology has played a very important role in current $3 \mathrm{G} / 4 \mathrm{G}$ wireless systems, massive MIMO and full-dimension MIMO present significant innovations for this technology to fundamentally increase the spectrum and energy efficiency of wireless systems [86]. More- over, massive MIMO can potentially simplify the ways we manage the radio resource allocation and cochannel interference in the multi-cell and multi-tier wireless net- work.

Many existing works design high energy-efficient massive MIMO systems. The number of users and transmit power were jointly optimized in [87]. The energy efficiency (EE) of massive MIMO systems depends greatly on the circuit power consumption. The number of antennas, users, and transmit power were respectively optimized for the single-cell massive MIMO system with zero-forcing beamforming (ZFBF) in [88]. The optimal transmit power increases with the number of antennas, which means that the transmit power is an important design parameter for high $\mathrm{EE}$ massive MIMO systems. In [89], the GEE considering both the uplink and downlink of a multi-cell massive MIMO system, subject to channel estimation errors, pilot contamination, and correlated channel paths, with either maximum ratio combining (MRC)/MRT or $\mathrm{ZF}$ transmission/reception is optimized Many existing designs for traditional MIMO systems must be re-thought for these new MIMO systems such as channel estimation, beamforming design, and signal detection. Moreover, there are also various arising challenges to resolve such as the channel estimation and pilot contamination issues, suitable multi-cell coordination, and hardware imperfection to name a few. Three papers published in our special issue address some of these research issues. Specifically, the work proposes a low-complexity sub- space detection technique for the multi-stream MIMO communications. In, a novel semi-orthogonal pilot design for massive MIMO wireless systems is developed to reduce the pilot resource consumption where a successive interference cancelation (SIC)based channel estimation is employed to manage the interference between data and pilot. Moreover, the work develops a joint channel coding, modulation, and MIMO communication scheme by using the rotated modulation and space-time component interleave.

Furthermore, the inter-cell coordination in multicell massive MIMO systems is addressed in various works, either suppressing the inter-cell interference and inter- user interference completely by coordinated beam- forming. Another technique introduced in allows interference suppression with smaller excess of base station antennas. Multi-cell MIMO down- link channel is studied and a distributed interference alignment (IA) algorithm is proposed to suppress or minimize the interference to non-intended users. Also, develops an IA technique for a downlink cellular system with CSI exchange and feedback within each cell. In, conditions for the feasibility of IA and degree of freedom (DoF) for MIMO cellular networks are investigated.

To reduce the complexity and CSI requirement, the concept of grouping-based IA (GIA) is proposed for a two- cell single-stream interfering MIMO-BC in [108]. This GIA is extended to a multi-cell interfering MIMO-BC in, where both the feasible condition on the GIA and a low complexity IA decoder design are studied. For the performance analysis of massive MIMO system, there exists a large set of tools from random matrix theory (see references therein).

\section{iv. C-RAN}

Cloud radio access network (C-RAN) presents a major paradigm shift in the design of future wireless systems where various network functionalities in both access and core networks can be realized in the cloud. Due to the high costefficiency, flexibility, and utilization efficiency, it is 
regarded as one of the most promising solutions to meet the huge capacity demand in $5 \mathrm{G}$ wireless networks. In a C-RAN, plenty of distributed remote radio heads (RRHs) are distributed within a specific geographical area and connected to a centralized base- band unit (BBU) pool through high bandwidth fronthaul links. In such a system, enhanced energy efficiency can be achieved from shortened distances between RRHs and users as well as improved spectral efficiency per unit area. Moreover, since all baseband processing are performed at the BBU, it is possible to employ CoMP transmission for capacity enhancement by exploiting the increased spatial degrees of freedom and beamforming gain. The paper in our special issue proposes a general $5 \mathrm{G}$ network architecture based on the C-RAN technology.

There are numerous open research issues to address in this interesting research domain including efficient radio resource management (e.g., power control, bandwidth allocation, beamforming designing), fronthaul compression, efficient design, and utilization of computing resources in the cloud to name a few. Some key open research issues are elaborated below.

\section{v. Joint RRH clustering and precoding}

To perform full CoMP among all RRHs, the BBU must exchange the baseband signals with all RRHs and collect the global CSI from all RRHs to all users. As the number of RRHs increases, the fronthaul loading and CSI signalling overhead will become unacceptable. More- over, from the system energy efficiency perspective, it is not necessary to turn on all the RRHs when the system loading is not heavy. This motivates dynamic clustering of RRHs into virtual base stations (VBSs) to serve users via partial CoMP. There are two different types of RRH clustering, namely the network centric clustering (NCC) and user centric clustering (UCC). In NCC, there is no overlap among VBSs (i.e., RRH can only belong to a single VBS). This can simplify the cluttering and precoding design, but the performance of the cell edge users at the boundary of the clusters will be compromised. In UCC, there can be overlap among VBSs, and we have the freedom to choose a small number of "best" RRHs as a VBS for each user to avoid cell edge effect. Both NCC and UCC can significantly reduce the fronthaul loading and CSI signalling overhead by controlling the size of each VBS. However, the UCC usually outperforms the NCC but the associated clustering and precoding design is more challenging. One side effect of RRH clustering is the inter-cluster interferences among different VBS. As a result, efficient joint $\mathrm{RRH}$ clustering and interference mitigation (precoding) schemes are essential for practical deployment of C-RAN. The existing joint clustering and precoding schemes can be classified into two classes.

One-timescale schemes: In such schemes, both RRH clustering and precoding (beamforming) are optimized at each time slot based on instantaneous global CSI. In, the authors developed an efficient cell-clustering algorithm to maximize the sum rate of the users based on graph theory. In, a joint clustering and scheduling algorithm is proposed to maximize the weighted sum rate by greedily selecting the BS clusters from a set of predetermined candidate clusters. A major approach to avoid the combinatorial optimization of RRH clustering is group sparse beamforming (GSBF), where the size of each RRH cluster (VBS) is reduced by imposing group sparsity constraints on the beamforming vectors such that the RRHs with zero beamforming vectors can be turned off. Various GSBF schemes have been proposed in based on the (weighted) mixed $l 1 / l p$-norm techniques with different objective functions and constraints. However, all of the above onetimescale schemes suffer from huge CSI signalling overhead and large computation complexity due to global centralized optimization. Moreover, it is difficult to obtain instantaneous global CSI in a CRAN with a large number of RRHs because of the 
signalling latency. Thus, these solutions are not scalable w.r.t. the network size.

Heuristic two-timescale schemes: In view of the challenges to implement the one-timescale schemes in practical C-RAN, some two-timescale schemes have been proposed where the RRH clustering is updated at slower timescale based on channel statistics, and the precoder is updated at each time slot based on instantaneous partial CSI from the active RRHs to the users. Since the number of active RRHs can be substantially smaller than the total number of RRHs in the network, such twotimescale design can significantly reduce the CSI signalling over- head and computational complexity. In, each user first chooses its serving BS cluster based on the channel statistics using the nearest base clustering and or the nearest interferer clustering. Then, the precoder is optimized using a soft interference nulling (SIN) precoding algorithm at each time slot. Liu and Lau studied the joint optimization of RRH selection, regularization factor in RZF precoding, and power allocation, where the $\mathrm{RRH}$ selection is adaptive to the global path gain matrix using a heuristic algorithm based on the deterministic equivalent of the average rate and power. In the existing two- timescale schemes, the $\mathrm{RRH}$ clustering and precoding solutions are obtained in a heuristic manner (i.e., the solution is not derived from a single joint optimization problem), and the performance gap of these heuristic solutions w.r.t. the optimal solution is still unknown.

One interesting future work in this direction is to develop an optimization based two-timescale joint $\mathrm{RRH}$ clustering and precoding scheme, where the long-term RRH clustering is adaptive to the channel statistics and the short-term precoding is adaptive to the instantaneous partial CSI from the active RRHs to the users. The optimization-based two-timescale solution gives implementation friendly and scalable solution because solutions of different timescales can be implemented at different hierarchies of $\mathrm{C}$ -
RAN and they are robust to signalling latency. It is also expected to achieve better trade- off between performance and implementation costs than the one-timescale scheme and heuristic two-timescale schemes. For example, similar two-timescale resource optimization schemes have been proposed in for massive MIMO systems or heterogeneous networks and they are shown to achieve much better trade-off between performance and various implementation costs. However, there are several technical challenges to achieve these good features of two-timescale resource optimization for C-RAN.

- Two-stage stochastic non-convex optimization: Due to the stochastic nature of the twotimescale optimization, there are usually expectation operators in the objective/constraint functions and the argument of the expectation operators involve the optimal short-term precoding solutions, which do not have closed form expressions. Moreover, the combinatorial nature of the RRH clustering optimization problem further complicates the problem.

- Lack of Channel Statistics: In practice, we might not even have the explicit knowledge of the channel statistics. The solution should be self-learning to the dynamic propagation environment.

\section{vi. Fronthaul loading compression/reduction}

In conventional C-RAN, the BBU pool and RRHs exchange the baseband signals via low latency fronthaul links in the form of digitized IQ samples. Such design requires prohibitive fronthaul capacities in typical set- tings, and the capacity of fronthaul links has become a performance bottleneck in C-RAN. Three major techniques have been proposed to reduce the fronthaul loading in the C-RAN: the RRH clustering which has already been reviewed above, the fronthaul compression, and the partially centralized C-RAN (PC-RAN). Various fronthaul compression schemes have been 
proposed based on vector quantization and possibly multivariate compression strategies. In the uplink, one can also exploit the sparsity of the baseband signals (caused by busty uplink traffic or random access) to further reduce the fronthaul loading using compressive sensing (CS). The CS-based fronthaul compression method in can only be applied to the case when the full CoMP is employed, and there is no inter-cluster interference. When partial CoMP is employed with RRH clustering, it is still possible to exploit the sparsity of the baseband signals and/or inter-cluster interference to do joint inter-cluster interference mitigation and fronthaul compression using the CS-based joint interference mitigation and detection algorithm in. Recently, proposed a PCRAN architecture where part of the simple baseband processing such as modulation and precoding is shifted into RRHs. By doing so, precoder, data symbols, and channel information are separately transported instead of heavy IQ data. As a result, the fronthaul loading can be significantly reduced with slightly more complex RRHs.

However, the existing fronthaul loading compression/reduction schemes treat the information bits sent to users as random bits and do not exploit the fact that con- tent (such as videos and music), which are cachable at the RRH, contributes to a large portion of today's wireless traffic. By caching some popular content at the RRHs, the RRHs may directly employ (partial) CoMP to transmit the cached content to the users without consuming the fronthaul. The BS-level PHY caching was first introduced in to exploit cached-induced opportunistic CoMP for cellular networks with limited backhaul capacity. In, the BS-level PHY caching is designed for cellular networks where all the baseband processing is performed at the BS. Hence, the BS can directly cache content packets to exploit cached-induced opportunistic CoMP. However, in C-RAN where all base- band processing is implemented in the BBU, the RRH can only cache IQ samples. Since the IQ samples of the same content packet will be different under different modulation and coding schemes (MCS), it is challenging to design an efficient RRH-level PHY caching scheme to reduce the fronthaul loading, which could be an interesting research topic.

\section{vii. Software defined networking and wireless virtualization}

Existing wireless cellular networks are based on standardized and inflexible hardware elements and network architecture, which hinder rapid deployment of new wireless applications, services, and adoption of emerging wireless technologies. It has been recognized that the SDN concept originally proposed for wired networks and data centres can be leveraged to engineer the $5 \mathrm{G}$ software defined wireless networks. In general, SDN aims to separate the control and data forwarding planes, which enables to achieve quick provisioning and reconfiguration as well as more efficient utilization of the network infrastructure. An SDN-based network is realized by allowing network hardware elements (e.g., switches) to be programmed and controlled through a standardized interface such as OpenFlow and by employing a network controller to make control decisions on the operations of the data forwarding infrastructure.

The network controller has a global view of the network by receiving updated traffic and network state information, which improves its decision-making efficiency. Moreover, network elements simply conduct required net- work functionality and protocols under the instruction of the centralized network controller. This saves a lot of time and resources and enables the operations and configuration of the multi-vendor network infrastructure to be realized more quickly and efficiently by software instead of custom configuration for many network elements deployed throughout the network. 
Most existing research on SDN has been conducted for wired networks, and there are very few works addressing the SDN-based design for wireless cellular networks. In fact, engineering the software defined wire- less cellular network involves to develop the network and control architecture covering both wireless access and core networks as well as the corresponding inter- faces. In addition, there is a strong demand in devising more flexible and efficient sharing of expensive wireless infrastructure among multiple (virtual) wireless service providers and operators using wireless virtualization technologies. Sharing of the wireless cellular infrastructure among multiple parties can be applied for both wireless access and core networks. This involves the sharing of different types of network resources including wireless spectrum and BSs, routers, and switches in the core network.

To realize such virtualization vision for wireless net- works, network resources must be abstracted and partitioned into different "slices", which can be then allocated to different (virtual) wireless operators who provide services to the end users. Development of such slicing mechanisms that guarantee efficient utilization of the network resources, satisfactory isolation among different slices to enable independent deployment of various network algorithms and protocols by the involved wireless operators and to provide QoS support for wireless users remains one major challenge to address in this interesting research theme. The SDN techniques can be lever- aged to implement efficient virtualization of the future wireless cellular networks where the SDN controller can play an important role in the slicing and allocation operations of resource slices and in the control and activation of different network algorithms and protocols deployed by the corresponding wireless operators associated with different resource slices.

Efficient design of the software defined wireless cellular network is challenging because of several reasons. First, the wireless access network has become more and more complex to meet the exponential growth of the mobile traffic, the diverse QoS and through- put requirements of different wireless applications and services, and the need to achieve more cost, energy, and spectrum efficient communications. Toward this end, engineering future wireless access networks follows some major trends including the evolution toward ultra-dense and multi-tiered deployment of different types of BSs (e.g., macro, micro, pico, and femto BSs), efficient utilization of the precious wireless spectrum in both microwave and mmWave bands using dynamic spectrum access and cognitive radio techniques, efficient coordination of radio resource management and transmission for better interference mitigation and spectrum utilization, development of advanced communication techniques such as massive and 3D MIMO, and employment of wireless access and backhaul communications. Therefore, optimization of the centralized access management for this complex wireless access presents a major challenge.

Development of an effective SDN architecture based on which control algorithms can be implemented for the software defined wireless cellular network is more challenging than that for the wired network. This is because the data forwarding plane in the wired network involves only routers, switches, and wired links with fixed capacity. In contrast, communication links in the wireless access network are strongly coupled since they share limited radio resources and simultaneous transmissions on one sub band create co-channel interference for each other. Moreover, modern wireless cellular networks are based on sophisticated network algorithms and protocols, which adapt their operations to various system dynamics such as time-varying wireless channel state and traffic information.

Therefore, there would be a huge amount of control signalling between the SDN controller and wireless 
data forward planes (mobile users and BSs) if a fully centralized control architecture is adopted in which the SDN controller makes all control decisions. To achieve a scalable and efficient architecture, it is likely that different SDN controllers for the wireless access and core networks must be deployed, which closely interact with each other. This would lead to desirable tradeoff between network performance and control signalling overhead and pro- vide more satisfactory end-to-end QoS performance for mobile users. Furthermore, the wireless access network may conduct various control functions which are based on highly dynamic system information such as wireless CSI in a semi-distributed manner using local controllers (e.g., employed at the BSs). Then, a well-designed net- work management framework can be developed so that these local controllers can efficiently interact with a centralized SDN controller, which makes more large-scale control decisions. For example, the CSI-driven wireless scheduling and radio resource management functions can be taken care by local controllers while load balancing and mobility control decisions can be realized by a centralized controller.

The control architecture for the core network of $4 \mathrm{G}$ and beyond wireless network could be established by leveraging significant advances on the SDN technology, which has been developed for the packet-switched networks. The fact that the core network of $4 \mathrm{G}$ wireless systems is based, the Evolved Packet Core (EPC) architecture established by the 3GPP [148] with well-separated control and data planes provides a suitable foundation for engineering this SDN architecture. Moreover, the SDN controller for the core network must be designed considering unique characteristics and requirements of different elements in the data forwarding plane such as mobility management, different service, and internet gateways. Furthermore, this design should be done so that it can support efficient sharing of the core network facility among multiple wire- less operators in the future virtualized wireless mobile network.

The overall control architecture and interfaces for the next-generation SDN-based wireless network should also be established to enable efficient integration of many advanced physical-layer wireless technologies, to allow effective QoS provisioning for diverse set of wireless applications with very different characteristics and requirements, to support very large numbers of access nodes and wireless connections, and to foster the emerging active infrastructure sharing among multiple wireless operators via virtualization techniques. Specifically, the northbound interfaces should be appropriately defined to allow active sharing of wireless access and/or core net- works among wireless operators and service providers where service providers can have sufficient power to control their traffic and optimize the assigned network resources. Moreover, the southbound interfaces should be engineered to achieve efficient utilization of net- work infrastructure and flexible programmable capability to provision and control the resource management and sharing operations under different RAN and wireless technologies.

An SDN architecture for the heterogeneous wireless dense network is proposed in, and another proposal of an SDN architecture for LTE-evolved packet core with design and analysis of five different procedures is dis- cussed in of our special issue. Development of efficient resource allocation solutions for future SDN-based wireless networks is the topic considered in two other papers of the special issue. Specifically, a three- step optimization framework to optimize the energy efficiency of the wireless network via adaptation of the user and control planes of the SDN-based wireless network is proposed in reference. Moreover, an SDN-based resource allocation and load balancing algorithm is developed to enhance the spectrum efficiency and users' QoS of the 
multi-tier and multi-RAT wireless network in reference.

There are still many open research challenges related to the development of different network and resource management and optimization tools to be deployed on the SDN control and interface architecture. Research on developing these important tools also has strong impacts on the design and tuning of the overall architecture since they are closely interacting elements of the software defined wireless cellular network. Examples of the tools to be developed are network and radio resource management, mobility and load balancing, traffic engineering, spectrum allocation and management, radio resource, and networking slicing tools. It is also worth mentioning that there are strong interactions and synergy between research on SDN and C-RAN for future $5 \mathrm{G}$ mobile wire- less systems since both technologies are based on the centralized optimization and management concepts of network resources and functionalities. In particular, data centres can be deployed to implement the SDN controller, which realizes various critical network management functions such as baseband processing, radio resource, mobility, and spectrum management. Obviously, there is much room for further research innovations as we evolve toward a unified, flexible, and efficient wireless ecosystem.

\section{CONCLUSION}

There have been some significant research advances in different 5G enabling wireless technologies and these research outcomes will form strong foundations for the $5 \mathrm{G}$ standardization activities, which are expected to start soon. Until then, it is desirable to develop a unified $5 \mathrm{G}$ architecture for wireless access and core networks that enable to integrate many advanced wireless technologies to meet the $5 \mathrm{G}$ requirements: realizing Gigabit, real- time, and ultra-reliable communications, supporting an extremely large number of wireless connections for both human and machine wireless applications, diverse set of mobile traffic with different characteristics, and QoS, and achieving more spectrum, energy, and costefficiency. There would be no single technology that can deliver these performance expectations. Therefore, a number of enabling $5 \mathrm{G}$ wireless technologies must be developed and jointly implemented on the newly developed 5G architecture. In this paper, we have reviewed the $5 \mathrm{G}$ requirements and discussed several key enabling $5 \mathrm{G}$ wireless technologies with their open research challenges. More- over, we have also described research papers in our special issue in the corresponding 5G network and technology contexts.

\section{REFERENCES}

[1]. Cisco, Cisco visual network index: Global mobile traffic forecast update 2014-2019. White paper (2015)

[2]. G Wu, S Talwar, K Johnsson, N Himayat, M2M: from mobile to embedded Internet. IEEE Commun. Mag. 49(4), 36-43 (2011)

[3]. N Bhushan, J Li, D Malladi, R Gilmore, D Brenner, A Damnjanovic, R Sukhavasi, C Patel, $S$ Geirhofer, Network densification: the dominant theme for wireless evolution into 5G. IEEE Commun. Mag. 52(2), 82-89 (2014)

[4]. F Boccardi, RW Heath, A Lozano, TL Marzetta, $\mathrm{P}$ Popovski, Five disruptive technology directions for 5G. IEEE Commun. Mag. 52(2), 74-80 (2014)

[5]. $\mathrm{R}$ Baldemair, $\mathrm{T}$ Irnich, $\mathrm{K}$ Balachandran, $\mathrm{E}$ Dahlman, G Mildh, Y Selen', S Parkvall, M Meyer, A Osseiran, Ultra-dense networks in millimeter-wave frequencies. IEEE Commun. Mag. 53(1), 202-208 (2015)

[6]. GP Fettweis, The tactile internet: applications and challenges. IEEE Veh. Technol. Mag. 9(1), 64-70 (2014) 
[7]. A Osseiran, F Boccardi, V Braun, K Kusume, P Marsch, M Maternia, O Queseth, M Schellmann, H Schotten, H Taoka, H Tullberg, MA Uusitalo, B Timus, M Fallgren, Scenarios for 5G mobile and wireless communications: the vision of the METIS project. IEEE Commun. Mag. 52(5), 26-35 (2014)

[8]. G Wunder, P Jung, M Kasparick, T Wild, F Schaich, Y Chen, S Brink, I Gaspar, N Michailow, A Festag, L Mendes, N Cassiau, D Ktenas, M Dryjanski, S Pietrzyk, B Eged, P Vago, F Wiedmann, 5GNOW: non-orthogonal, asynchronous waveforms for future mobile applications. IEEE Commun. Mag. 52(2), 97-105 (2014)

[9]. B Farhang-Boroujeny, OFDM versus filter bank multicarrier. IEEE Signal Process Mag. 28(3), 92-112 (2011)

[10]. Z Pi, F Khan, An introduction to millimeterwave mobile broadband systems. IEEE Commun. Mag. 49(6), 101-107 (2011)

[11]. S TS Rappaport, R Sun, H Mayzus, Y Zhao, K Azar, GN Wang, JK Wong,

[12]. M Schulz, F Samimi, Gutierrez, Millimeter wave mobile communications for $5 \mathrm{G}$ cellular: it will work. IEEE Access. 1, 335-349 (2013)

[13]. JG Andrews, What will 5G be. IEEE J. Sel. Areas Commun. 32(6), 1065-1082 (2014)

[14]. V Chandrasekhar, JG Andrews, A Gatherer, Femtocell networks: a survey. IEEE Commun. Mag. 46(9), 59-67 (2008)

[15]. MN Tehrani, M Uysal, $\mathrm{H}$ Yanikomeroglu, Device-to-device communication in 5G cellular networks: challenges, solutions, and future directions. IEEE Commun. Mag. 52(5 ), 86-92 (2014)

[16]. N NaderiAlizadeh, S Avestimehr, ITLinQ: a new approach for spectrum sharing in device-todevice communication systems. IEEE J. Sel. Areas Commun. 32(6), 1573-1577 (2014)

[17]. F Rusek, D Persson, BK Lau, E Larsson, T Marzetta, O Edfors, F Tufvesson, Scaling up
MIMO: opportunities and challenges with very large arrays. IEEE Signal Process. Mag. 30(1), 40-60 (2013)

[18]. Y-H Nam, BL Ng, K Sayana, Y Li, J Zhang, Y Kim, J Lee, Full-dimension, MIMO (FD-MIMO) for next generation cellular technology. IEEE Commun. Mag. 51(6), 172-179 (2013)

[19]. A Kammoun, H Khanfir, Z Altman, M Debbah, M Kamoun, Preliminary results on 3D channel modeling: from theory to standardization. IEEE J. Sel. Areas Commun. 32(6), 1219-1229 (2014)

[20]. P Rost, CJ Bernardos, AD Domenico, MD Girolamo, M Lalam, A Maeder, D Sabella, D Wuibben, Cloud technologies for flexible $5 \mathrm{G}$ radio access networks. IEEE Commun. Mag. 52(5), 68-76 (2014)

[21]. S Sezer, Are we ready for SDN? Implementation challenges for software-defined networks. IEEE Commun. Mag, 36-43 (2013)

[22]. C Liang, FR Yu, Wireless network virtualization: a survey, some research issues and challenges. IEEE Commun. Surveys \& Tutorials. 17(1), 358-380 (2014)

[23]. E Bjornson, E Jorswieck, M Debbah, B Ottersten, Multi-objective signal processing optimization: the way to balance conflicting metrics in $5 G$ systems. IEEE Signal Process. Mag. 31(6), 14-23 (2014)

[24]. S Sun, TS Rappaport, Heath Jr. RW, A Nix, Rangan S, MIMO for millimeter-wave wireless communications: beamforming, spatial multiplexing, or both? IEEE Commun. Mag. 52(12), 110-121 (2014)

[25]. JF Monserrat, G Mange, V Braun, H Tullberg, G Zimmermann, 'O Bulakci, METIS research advances towards the $5 \mathrm{G}$ mobile and wireless mobile system definition. EURASIP J. Wirel. Commun. Netw, 53 (2015)

[26]. A Chiumento, M Bennis, C Desset, L der Perre, S Pollin, Adaptive CSI and feedback estimation in LTE and beyond: a Gaussian process 
regression approach. EURASIP J. Wirel. Commun. Netw. 2015, 168 (2015)

[27]. X Yin, C Ling, M-D Kim, H Chung, Parameter estimation using the sliding-correlator's output for wideband propagation channels. EURASIP J. Wirel. Commun. Netw. 2015, 165 (2015)

[28]. Y Shibata, N Yoda, T Ohtsuki, J Mashino, T Sugiyama, Iterative estimation of undesired signal power for superposed multicarrier transmission with channel estimation error. EURASIP J. Wirel. Commun. Netw. 2015, 23 (2015)

[29]. Z Liu, W Xu, S Li, J Lin, Network-coded primary-secondary cooperation in OFDM-based cognitive multicast networks. EURASIP J. Wirel. Commun. Netw. 2015, 144 (2015)

[30]. C Ling, X Yin, S Boqué, M García-Lozano, Power minimization of cooperative beamforming networks with spectrum sharing. EURASIP J. Wirel. Commun. Netw. 2015, 72 (2015)

[31]. A Barbieri, D Fertonani, G Colavolpe, Timefrequency packing for linear modulations: spectral efficiency and practical detection schemes. IEEE Trans. Commun. 57(10), 29512959 (2009)

[32]. F Rusek, J Anderson, Multistream faster than Nyquist signaling. IEEE Trans. Commun. 57(5), 1329-1340 (2009)

[33]. J Anderson, F Rusek, V Owall, Faster-thanNyquist signaling. Proc. IEEE. 101(8), 18171830 (2013)

[34]. MJ Abdoli, M Jia, in Proc. IEEE VTC-Fall. Trellis decoding for multi-user faster-thanNyquist transmission, (2014)

[35]. N Michailow, Matthé M, IS Gaspar, AN Caldevilla, LL Mendes, A Festag, G Fettweis, Generalized frequency division multiplexing for 5th generation cellular networks. IEEE Trans. Commun. 62(9), 3045-3061 (2010)

[36]. G Fettweis, M Krondorf, S Bittner, in Proc. IEEE Vehicular Technology Conference.
GFDM: generalized frequency division multiplexing, (2009), pp. 1-4

[37]. D Dasalukunte, F Rusek, V wall, Multicarrier faster-than-Nyquist signaling transceivers: hardware architecture and performance analysis. IEEE Trans. Circ. Syst. I, Reg. Papers. 58(4), 827-838 (2011)

[38]. MJ Abdoli, M Jia, J Ma, in Proc. IEEE PIMRC. Weighted circularly convolved filtering in OFDM/OQAM, (2013)

[39]. M Caus, AI Perez-Neira, Multi-stream transmission for highly frequency selective channels in MIMO-FBMC/OQAM systems. IEEE Trans. Signal Process. 62(4), 786-796 (2014)

[40]. M Caus, AI Perez-Neira, Transmitter-receiver designs for highly frequency selective channels in MIMO FBMC systems. IEEE Trans. Signal Process. 60(12), 6519-6532 (2012)

[41]. V Vakilian, T Wild, F Schaich, Brink St, J-F Frigon, in Proc. 9th International Workshop on Broadband Wireless Access, IEEE Globecom. Universal filtered multi-carrier technique for wireless systems beyond LTE, (2013)

[42]. T Wild, F Schaich, Y Chen, in Proc. Int. Conf. Dig. Signal Process. (DSP). 5G air interface design based on universal filtered (UF-) OFDM, (2014),pp. 699-704

[43]. J Abdoli, M Jia, J Ma, in Proc. IEEE Int'l Workshop on Signal Processing Advances in Wireless Communications (SPAWC). Filtered OFDM: a new waveform for future wireless systems, (2015)

[44]. M Matthe, LL Mendes, I Gaspar, N Michailow, D Zhang, G Fettweis, Multi-user time-reversal STC-GFDMA for future wireless networks. EURASIP J. Wirel. Commun. Netw. 2015, 132 (2015)

[45]. K Higuchi, Y Kishiyama, in Proc. APWCS 2012. Non-orthogonal access with successive interference cancellation for future radio access, (2012) 
[46]. H Nikopour, H Baligh, in Proc. IEEE PIMRC. Sparse code multiple access, (2013)

[47]. M Taherzadeh, H Nikopour, A Bayesteh, H Baligh, in Proc. IEEE VTC-Fall. CMA codebook design, (2014)

[48]. SN Premnath, D Wasden, SK Kasera, N Patwari, B Farhang-Boroujeny, Beyond OFDM: besteffort dynamic spectrum access using filterbank multicarrier. IEEE/ACM Trans. Netw. 21(3), 869-882 (2013)

[49]. HS Dhillon, RK Ganti, F Baccelli, JG Andrews, Modeling and analysis of K-tier downlink heterogeneous cellular networks. IEEE J. Sel. Areas Commun. 30(3), 55060 (2012)

[50]. V Chandrasekhar, JG Andrews, A gatherer femtocell networks: a survey. IEEE Commun. Mag. 46(9), 59-67 (2008)

[51]. N Saquib, E Hossain, LB Le, DI Kim, Interference management in OFDMA femtocell networks: issues and approaches. IEEE Wirel. Commun. 19(3), 86-95 (2012)

[52]. LB Le, D Niyato, E Hossain, DI Kim, DT Hoang, QoS-aware and energy-efficient resource management in OFDMA femtocells. IEEE Trans. Wirel. Commun. 12(1), 180-194 (2013)

[53]. Q Ye, B Rong, Y Chen, M Al-Shalash, C Caramanis, JG Andrews, User association for load balancing in heterogeneous cellular networks. IEEE Trans. Wirel. Commun. 12(6), 2706-2716 (2013)

[54]. K Shen, W Yu, Distributed pricing-based user association for downlink heterogeneous cellular networks. IEEE J. Sel. Areas Commun. 32(6), 1100-1113 (2014)

[55]. VN Ha, LB Le, Distributed base station association and power control for heterogeneous cellular networks. IEEE Trans. Vehicular Technol. 63(1), 282-296 (2014)

[56]. S Singh, JG Andrews, Joint resource partitioning and offloading in heterogeneous cellular networks. IEEE Trans. Wirel. Commun. 13(2), 888-901 (2014)
[57]. S Deb, P Monogioudis, J Miernik, JP Seymour, Algorithms for enhanced inter-cell interference coordination (eICIC) in LTE HetNets. IEEE/ACM Trans. Netw. 22(1), 13750 (2014)

[58]. E Hossain, LB Le, D Niyato, Radio Resource Management in Multi-Tier Cellular Wireless Networks. (Wiley, New York, NY, USA, 2013)

[59]. D Lopez-Perez, D Lopez-Perez, I Guvenc, G de la Roche, M Kountouris, TQS Quek, J Zhang, Enhanced intercell interference coordination challenges in heterogeneous networks. IEEE Wirel. Commun. 18(3), 22-30 (2011)

[60]. D Nguyen, LB Le, $\mathrm{T}$ Le-Ngoc, Multiuser admission control and beamforming optimization algorithms for MISO heterogeneous networks. IEEE Access. 3, 759773 (2015)

[61]. VN Ha, LB Le, Fair resource allocation for OFDMA femtocell networks with macrocell protection. IEEE Trans. Vehicular Technol. 63(3), 1388-1401 (2014)

[62]. DT Ngo, LB Le, T Le-Ngoc, Distributed Paretooptimal power control for utility maximization in femtocell networks. IEEE Trans. Wirel. Commun. 11(10), 3434-3446 (2012)

[63]. DT Ngo, LB Le, T Le-Ngoc, E Hossain, DI Kim, Distributed interference management in twotier CDMA femtocell networks. IEEE Trans. Wirel. Commun. 11(3), 979-989 (2012)

[64]. FH Panahi, T Ohtsuki, Stochastic geometry modeling and analysis of cognitive heterogeneous cellular networks. EURASIP J. Wirel. Commun. Networking. 2015, 141 (2015)

[65]. Q Zhang, T Yang, Y Zhang, Z Feng, Fairness guaranteed novel eICIC technology for capacity enhancement in multi-tier heterogeneous cellular networks. EURASIP J. Wirel. Commun. Networking. 2015, 62 (2015)

[66]. Y Sui, I Guvenc, $\mathrm{T}$ Svensson, Interference management for moving networks in ultradense urban scenarios. EURASIP J. Wirel. Commun. Networking. 2015, 111 (2015) 
[67]. TD Hoang, LB Le, T Le-Ngoc, in Proc. IEEE GLOBECOM. Dual decomposition method for energy-efficient resource allocation in D2D communications underlying cellular networks, (2015)

[68]. TD Hoang, LB Le, T Le-Ngoc, in Proc. IEEE ICC. Energy-efficient resource allocation for D2D communications in cellular networks, (2015)

[69]. TD Hoang, LB Le, T Le-Ngoc, in Proc. IEEE GLOBECOM. Resource allocation for D2D communications under proportional fairness, (2014)

[70]. LB Le, in Proc. IEEE GLOBECOM. Fair resource allocation for device-to-device communications in wireless cellular networks, (2012)

[71]. Q Wang, C Lai, Y Dong, XXu Y Shu, Joint user clustering and resource allocation for device-todevice communication underlaying MU-MIMO cellular networks. EURASIP J. Wirel. Commun. Networking. 2015, 145 (2015)

[72]. S Shah, J Gu, S Hasan, M Chung, SC-FDMAbased resource allocation and power control scheme for D2D communication using LTE-A uplink resource. EURASIP J. Wirel. Commun. Networking. 2015, 137 (2015)

[73]. W Roh, J-Y Seol, J Park, B Lee, J Lee, Y Kim, J Cho, K Cheun, F Aryanfar, Millimeter-wave beamforming as an enabling technology for 5G cellular communications: theoretical feasibility and prototype results. IEEE Commun. Mag. 52(2), 106-113 (2014)

[74]. T Rappaport, F Gutierrez, E Ben-Dor, J Murdock, Y Qiao, J Tamir, Broadband millimeter-wave propagation measurements and models using adaptive-beam antennas for outdoor urban cellular communications. IEEE Trans. Antennas Propag. 61(4), 1850-1859 (2013)

[75]. Mathias C, Fixing the cellular network: backhaul is the key (2008). http:// www.networkworld.com/community/print/359
20. Accessed Dec 2008 It all comes back to backhaul, Ericsson white paper (2014). http://www.

ericsson.com/res/docs/whitepapers/WP-

Heterogeneous- Networks- Backhaul.pdf. Accessed Sep 2015

[76]. S Chia, M Gasparroni, P Brick, The next challenge for cellular networks: backhaul. IEEE Microwave Mag. 10(5), 54-66 (2009)

[77]. TS Rappaport, JN Murdock, F Gutierrez, State of the art in $60-\mathrm{GHz}$ integrated circuits and systems for wireless communications. Proc. IEEE. 99(8), 1390-1436 (2011)

[78]. H Zhang, S Venkateswaran, U Madhow, in Proc. IEEE Globecom. Analog multitone with interference suppression: relieving the ADC bottleneck for wideband $60 \mathrm{Ghz}$ systems, (2012)

[79]. S O El Ayach, S Rajagopal, Z Abu-Surra, R Pi, Heath, Spatially sparse precoding in millimeter wave MIMO systems. IEEE Trans. Wirel. Commun. 13(3), 1499-1513 (2014)

[80]. A Alkhateeb, O El Ayach, G Leus, R Heath, Channel estimation and hybrid precoding for millimeter wave cellular systems. IEEE J. Sel. Topics Signal Process. 8(5), 831-846 (2014)

[81]. S Hur, T Kim, DJ Love, JV Krogmeier, TA Thomas, A Ghosh, Millimeter wave beamforming for wireless backhaul and access in small cell networks. IEEE Trans. Commun. 61(10), 4391-4403 (2013)

[82]. T Bogale, LB Le, A Haghighat, in Proc. IEEE ICC. User scheduling for massive MIMO OFDMA systems with hybrid analog-digital beamforming, (2015) T Bogale, LB Le, in Proc. IEEE GLOBECOM. Beamforming for multiuser massive MIMO systems: digital versus hybrid analog-digital, (2014)

[83]. H Ishii, Y Kishiyama, $\mathrm{H}$ Takahashi, in Proc. Globecom Workshops. A novel architecture for LTE-B: C-plane/U-plane split and phantom cell concept, (2012) 
[84]. Q Li, H Niu, G Wu, RQ Hu, in Proc. IEEE Globecom Workshop. Anchor-booster based heterogeneous networks with mmWave capable booster cells, (2013)

[85]. T Marzetta, Noncooperative cellular wireless with unlimited numbers of base station antennas. IEEE Trans. Wirel. Commun. 9(11), 3590-600 (2010)

[86]. H Yang, TL Marzetta, in Proc. IEEE GreenCom. Total energy efficiency of cellular large scale antenna system multiple access mobile networks, (2013)

[87]. E Bjönson, L Sanguinetti, J Hoydis, M Debbah, Optimal design of energy-efficient multi-user MIMO systems: is massive MIMO the answer? IEEE Trans. Wirel. Commun. 14(2), 3059-3075 (2015)

[88]. W Liu, A Zappone, C Yang, E Jorswieck, in Proc IEEE SPAWC. Global EE optimization of massive MIMO systems, (2015)

[89]. J Hoydis, S ten Brink, M Debbah, Massive MIMO in the UL/DL of cellular networks: how many antennas do we need. IEEE J.Sel. Areas Commun. 31(2), 160-171 (2013)

[90]. H Yang, TL Marzetta, Performance of conjugate and zero-forcing beamforming in large-scale antenna systems. IEEE J. Sel. Areas Commun. 31(2), 172-179 (2013)

[91]. X Rao, VKN Lau, Distributed compressive CSIT estimation and feedback for FDD multi-user massive MIMO systems. IEEE Trans. Signal Process. 62(12), 3261-3271 (2014)

[92]. J Chen, VKN Lau, Two-tier precoding for FDD multi-cell massive MIMO time-varying interference networks. IEEE J. Sel. Areas Commun. 32(6), 1230-1238 (2014)

[93]. T Bogale, LB Le, X Wang, L Vandendorpe, in Proc. IEEE GLOBECOM. Pilot contamination mitigation for wideband massive MMO: number of cells vs multipath, (2015)

[94]. H Yin, D Gesbert, M Filippou, Y Liu, A coordinated approach to channel estimation in large-scale multiple-antenna systems. IEEE J. Sel. Areas Commun. 31(2), 264-273 (2013)

[95]. B Dai, W Yu, Sparse beamforming and usercentric clustering for downlink cloud radio access network. IEEE Access. 2, 1326-1339 (2014)

[96]. 122. J-M Moon, D-H Cho, in Proc. IEEE 73rd Vehicular Technology Conference (VTC Spring). Inter-cluster interference management based on cell-clustering in network MIMO systems, (2011), pp. 1-6

[97]. 123. P Baracca, F Boccardi, V Braun, in Proc International Symposium on Wireless Communication Systems (ISWCS). A dynamic joint clustering scheduling algorithm for downlink CoMP systems with limited CSI, (2012), pp. 830-834

[98]. 124. M Hong, R Sun, H Baligh, Z-Q Luo, Joint base station clustering and beamformer design for partial coordinated transmission in heterogeneous networks. IEEE J. Select. Areas Commun. 31(2), 226-240 (2013)

[99]. 125. Y Shi, J Zhang, K Letaief, Group sparse beamforming for green cloud-ran. IEEE Trans. Wirel. Commun. 13(5), 2809-2823 (2014)

[100]. 126. B Dai, W Yu, in IEEE GLOBECOM. Sparse beamforming for limited-backhaul network MIMO system via reweighted power minimization, (2013), pp. 1962-1967

[101].127. C Ng, $\mathrm{H}$ Huang, Linear precoding in cooperative MIMO cellular networks with limited coordination clusters. IEEE J. Select. Areas Commun. 28(9), 1446-1454 (2010)

[102]. A Liu, V Lau, Joint power and antenna selection optimization in large cloud radio access networks. IEEE Trans. Signal Process. 62(5), 1319-1328 (2014)

[103]. S Wagner, R Couillet, M Debbah, DTM Slock, Large system analysis of linear precoding in correlated MISO broadcast channels under limited feedback. IEEE Trans. Inform. Theory. 58(7), 4509-4537 (2012) 
[104]. A Liu, V Lau, Hierarchical interference mitigation for massive MIMO cellular networks. IEEE Trans. Signal Process. 62(18), 4786-4797 (2014)

[105]. A Liu, V Lau, L Ruan, J Chen, D Xiao, Hierarchical radio resource optimization for heterogeneous networks with enhanced intercell interference coordination (eICIC). IEEE Trans. Signal Process. 62(7), 1684-1693 (2014)

[106]. A Liu, VKN Lau, Phase only RF precoding for massive MIMO systems with limited RF chains. IEEE Trans. Signal Process. 62(17), 4505-4515 (2014)

[107]. M Peng, C Wang, V Lau, H Poor, Fronthaulconstrained cloud radio access networks: insights and challenges. IEEE Wirel. Commun. 22(2), 152-160 (2015)

[108]. S-H Park, O Simeone, O Sahin, S Shamai, Joint precoding and multivariate backhaul compression for the downlink of cloud

[109]. radio access networks. IEEE Trans. Signal. Process. 61(22), 5646-5658 (2013)

[110]. SH Park, O Simeone, O Sahin, S Shamai, Joint decompression and decoding for cloud radio access networks. IEEE Signal Process. Letters. 20(5), 503-506 (2013)

[111]. P Patil, W Yu, in Proc. Information Theory and Applications Workshop (ITA). Hybrid compression and message-sharing strategy for the downlink cloud radio-access network, (2014), pp. 1-6

[112].X Rao, V Lau, Distributed fronthaul compression and joint signal recovery in CloudRAN. IEEE Trans. Signal Process. 63(4), 10561065 (2015)

[113]. A Liu, V Lau, Joint interference mitigation and data recovery in compressive domain: a sparse MLE approach. IEEE Trans. Signal Process. 62(19), 5184-5195 (2014)

[114]. S Park, C-B Chae, S Bahk, Large-scale antenna operation in heterogeneous cloud radio access networks: a partial centralization approach
(2015). [Online] Available: http://arxiv.org/abs/1405.2295. Accessed Sep 2015

[115]. A Liu, V Lau, Cache-enabled opportunistic cooperative MIMO for video streaming in wireless systems. IEEE Trans. Signal Process. 62(2), 390-402 (2014)

[116]. A Lui, UKN Lau, Mixed-timescale precoding and cache control in cached MIMO interference network. IEEE Trans. Signal Process. 61(24), 6320-6332 (2013)

[117]. BAA Nunes, M Mendonca, X-N Nguyen, K Obraczka, T Turletti, A survey of softwaredefined networking: past, present, and future of programmable networks. IEEE Commun. Surveys \& Tutorials. 16(3), 1617-1634 (2014)

[118]. N McKeown, Openflow: enabling innovation in campus networks. SIGCOMM Comput. Commun. Rev. 38(2), 69-74 (2008)

[119].H Kim, N Feamster, Improving network management with software defined networking. IEEE Commun. Mag. 51(2), 114-119 (2013)

[120]. F Hu, Q Hao, K Bao, A survey on softwaredefined network and openFlow: from concept to implementation. IEEE Commun. Surveys \& Tutorials. 16(4), 2181-2206 (2014)

[121]. Y Jarraya, T Madi, M Debbabi, A survey and a layered taxonomy of software-defined networking. IEEE Commun. Surveys \& Tutorials. 16(4), 1955-1980 (2014)

[122]. A Gudipati, D Perry, LE Li, S Katti, in Proc. ACM SIGCOMM workshop on Hot topics in software defined networking. SoftRAN: software defined radio access network, (2013)

[123]. K Pentikousis, Y Wang, W Hu, Mobileflow: toward software-defined mobile networks. IEEE Commun. Mag. 51(7), 44-53 (2013)

[124]. CJ Bernardos, A De La Oliva, P Serrano, A Banchs, LM Contreras, H Jin, JC Zun' iga, An architecture for software defined wireless networking. IEEE Wirel. Commun. 21(3), 52-61 (2014) 
[125]. H Farmanbar, H Zhang, in Prof. IEEE Network Operations and Management Symposium (NOMS). Traffic engineering for softwaredefined radio access networks, (2014)

[126]. N-D Dao, H Zhang, X Li, P Leroux, in Proc. IEEE Int'l Conf. Computing, Networking and Communications (ICNC). Radio access network coordination framework toward 5G mobile wireless networks, (2015)

[127]. NMMK Chowdhury, R Boutaba, A survey of network virtualization. Comput. Net. 54(5), 862-876 (2010)

[128]. R Kokku, R Mahindra, H Zhang, S Rangarajan, NVS: a substrate for virtualizing wireless resources in cellular networks. IEEE/ACM Trans. Networking. 20(5), 1333-1346 (2012)

[129]. F Fu, UC Kozat, in Proc. INFOCOM. Wireless network virtualization as a sequential auction game, (2010)

[130]. X Costa-Pérez, J Swetina, T Guo, R Mahindra, S Rangarajan, Radio access network virtualization for future mobile carrier networks. IEEE Commun. Mag. 51(7), 27-35 (2013)

[131]. M Kamel, LB Le, A Girard, in IEEE VTC-Fall. LTE wireless network virtualization: dynamic slicing via flexible scheduling, (2014)

[132]. M Kamel, LB Le, A Girard, in Proc. IEEE WCNC. LTE multi-cell dynamic resource allocation for wireless network virtualization, (2015)

[133]. JS Panchal, RD Yates, MM Buddhikot, Mobile network resource sharing options: performance comparisons. IEEE Trans. Wirel. Commun. 12(9), 4470-4482 (2013)

[134].H Zhang, S Vrzic, G Senarath, ND Dao, H Farmanbar, J Rao, C Peng, H Zhuang, 5G wireless network: MyNET and SONAC. IEEE Netw. 29(4), 14-23 (2015)

[135]. VM Borges, K Cardoso, E Cerqueira, M Nogueira, A Santos, Aspirations, challenges, and open issues for software-based $5 \mathrm{G}$ networks in extremely dense and heterogeneous scenarios.
EURASIP J. Wirel. Commun. Networking. 2015, 164 (2015)

[136]. VG Nguyen, Y Kim, Proposal and evaluation of SDN-based mobile packet core networks. EURASIP J. Wirel. Commun. Networking. 2015, 172 (2015)

[137]. X Xu, X Dai, Y Liu, R Gao, X Tao, Energy efficiency optimization-oriented control plane and user plane adaptation with a frameless network architecture for 5G. EURASIP J. Wirel. Commun. Networking. 159(2015) (2015)

[138]. X Duan, AM Akhtar, X Wang, Software-defined networking-based resource management: data offloading with load balancing in 5G HetNet. EURASIP J. Wirel. Commun. Networking. 2015, 181 (2015)

\section{Cite this article as :}

Dr. Hitesh H Vandra, "5G Technology : A Survey of Architecture and Emerging Technologies", International Journal of Scientific Research in Science and Technology (IJSRST), Online ISSN : 2395-602X, Print ISSN : 2395-6011, Volume 2 Issue 6 , pp. 642645, November-December 2016. Available at doi $\quad$ : https://doi.org/10.32628/IJSRST21830 Journal URL : https://ijsrst.com/IJSRST21830 